\title{
CAD-CAM Occlusal Splints: Milling and Printing Methods
}

\begin{abstract}
SMARANDA BUDURU ${ }^{1}$, DANIEL TALMACEANU2*, OANA BARU², RARES BUDURU³, CAMELIA SZUHANEK ${ }^{4}$, ANCA MESAROS 5
${ }^{1}$ luliu Hatieganu University of Medicine and Pharmacy, Department of Prosthodontics, 32 Clinicilor Str., 40006, Cluj Napoca, Romania

${ }^{2}$ Iuliu Hatieganu University of Medicine and Pharmacy, 32 Clinicilor Str., 40006, Cluj Napoca, Romania

35tomestet Dental Clinic, 68 A Calea Manastur, 400372,Cluj Napoca, Romania

${ }^{4}$ Victor Babes University of Medicine and Pharmacy Timisoara, Faculty of Dental Medicine, 2 Eftimie Murgu Sq., 300041, Timisoara, Romania

5/uliu Hatieganu University of Medicine and Pharmacy, Department of Dental Propaedeutics and Aesthetics, 32 Clinicilor Str., 40006, Cluj Napoca, Romania

In dentistry, splints are often used as means of protecting teeth, ceramic restorations or for treating temporomandibular dysfunction. Digital dentistry has become a common tool involving the following: intraoral scanners, model scanners, design software, computerized milling or printing. Our study aims at comparing two different methods of producing an occlusal splint (milled and printed) with the end result of determining the most suitable solution for patients in terms of time, costs, efficiency, and medical results.
\end{abstract}

Keywords: CAD CAM, milling, 3D printing, intraoral scanner, occlusal splint

Bite splints (occlusal appliances) are often seen by practitioners as a simple tool to protect teeth from wear or to protect the restorations. In fact, the use of these appliances can have manifold reasons: diagnosing the etiology of pain, treating muscle or temporomandibular joint (TMJ) pain, testing a new occlusal position, obtaining a seated condylar position, protecting teeth or restoration, treating sleep apnea. Different splints can be used depending on their role: anterior bite splints, full coverage splints, posterior appliances, mandibular repositioning splints, soft splints [1-5].

In the era of digital dentistry, occlusal appliances can be fabricated using CAD CAM techniques. The aim of this article is to evaluate the digital process involved in creating occlusal splints and investigate the advantages of digital bite splints [6,7]

\section{Experimental part}

Materials and methods

For this study, a patient diagnosed with temporomandibular disorders (TMD) was taken into consideration. The patient showed muscle tension, but no TMJ sign of dysfunction. The starting point in the patient's treatment was bite splint therapy in order to obtain muscle relaxation and musculoskeletal stable position. The steps of fabricating the digital bite splint are described next. Computerized axiography (Cadiax 2 Compact, Gamma Dental, Vienna, Austria) was performed in order to obtained the numeric values of sagittal condylar path and Bennett angle inclination.

\section{Clinical steps}

First, an optic impression was taken for the upper arch, lower arch and the occlusion was recorded (fig. 1). This process was done using the 3Shape Trios intraoral scanner. At the same time, a polyvinyl siloxane impression technique was done to try-in the splint after the digital fabrication. A facebow was recorded (Artex, AmannGirrbach) and the models were mounted in the semiadjustable articulator.

\section{Technical steps}

After the scan, the design of the digital splint was made using 3Shape Dental Designer-Appliance Designer

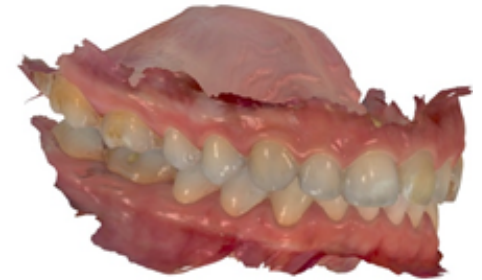

Fig. 1 Intraoral scan of the upper and lower jaw and occlusion

software. The first step was to block the undercuts and to define the insertion direction and the limits of the splint (fig. 2).

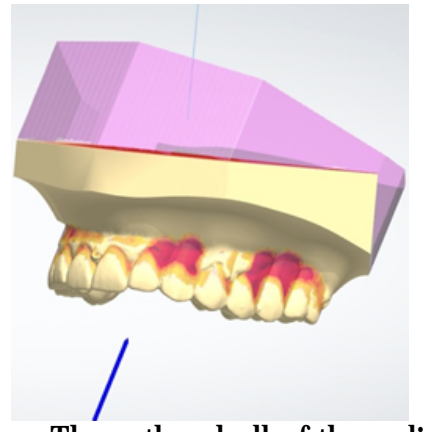

Fig. 2 Blocking the undercuts and defining the insertion

Then, the shell of the splint was designed, ensuring at least $1 \mathrm{~mm}$ thickness (fig. 3 ). In order to design an anatomical splint, a splint bar was designed (fig. 4). The soft recommended having at least $3 \mathrm{~mm}$ of total thickness in order to be able to adjust the splint in occlusion.

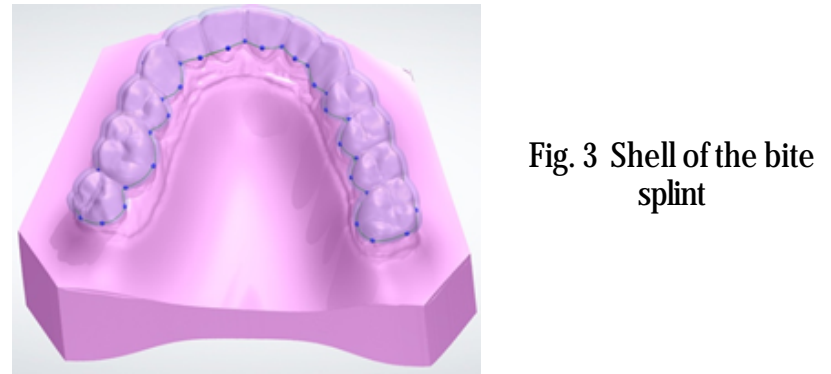

After the design of the splint was ready, the occlusion was checked in the virtual articulator, ensuring canine guidance and protrusion guidance (fig. 5). The values of the articulator were set with the sagittal condylar inclination and Bennett Angle of the patient.

For the milled splint we used a polymethylmethacrylate (PMMA) disc (Imes-Icore) and a Wieland Zenotec Select 


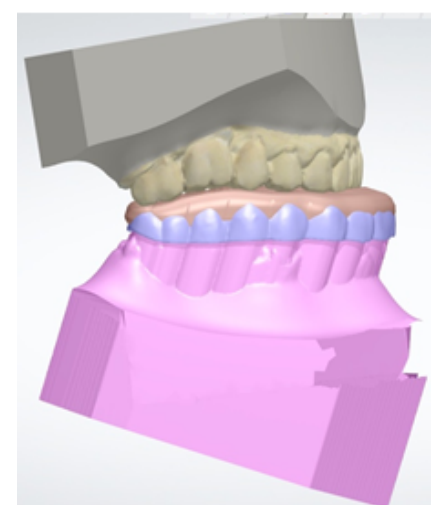

Fig.4 Adding the splintbar

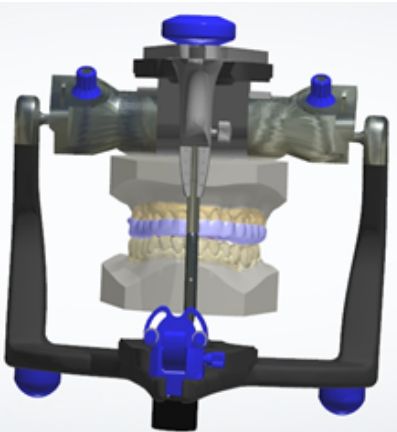

Fig. 5Adjusting the occlusion in the virtual articulator

milling machine. For the printed splint we used Dental LT Clear Resin (Formlabs) and the printer used was Form 2 (Formlabs).

\section{Materials}

For the milled splint, a polymethylmethacrylate (PMMA) Inno Blanc Coritec Disc (IMES-ICORE) disc was used. PMMA is an acrylic resin and its chemical formula is $\left(\mathrm{C}_{5} \mathrm{O}_{2} \mathrm{H}_{8}\right)_{n}$ Acrylic resins are the result of a free radical addition polymerization chain reaction. When a double bond is opened, another free radical available to attach another double bond is formed and so on and so forth ( fig. 6) [8-10].

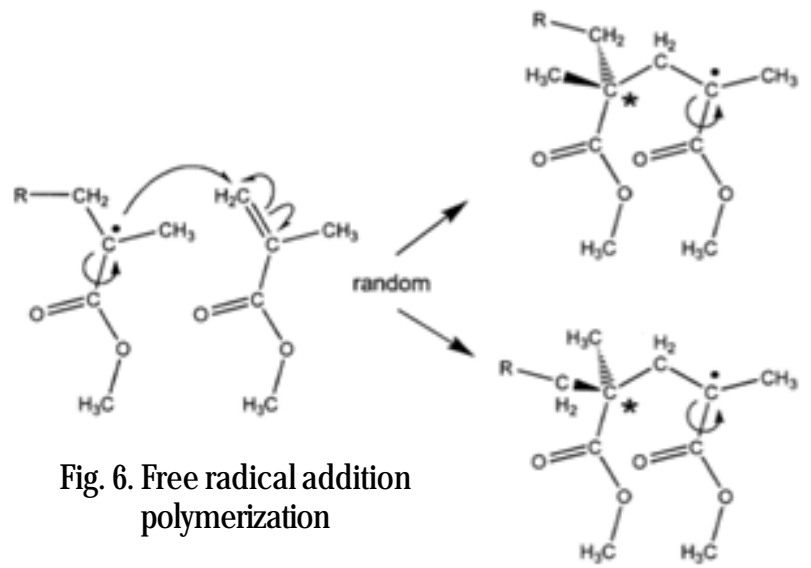

The properties of Inno Blanc Coritec PMMA splint are described in table 1.

\begin{tabular}{|l|}
\hline Residual monomer content $0.19 \%$ \\
\hline Cell vitality rate $96 \%-98 \%$ \\
\hline $\begin{array}{l}\text { Thermoplastic acrylic polymer based } \\
\text { on methyl methacrylate }\end{array}$ \\
\hline Without toxic or allergenic substances \\
\hline
\end{tabular}

Table 1 PROPERTIESOF THE INNO BLANC CORITEC PMMADISC

A class II long-term biocompatible resin (Dental LT Clear, Formlabs) with high resistance to fracture and wear was used for the 3D printed splint. Class II resin is an SLA (stereolithography) material. These materials are biocompatible and can come in contact with the human body (body fluids or open wounds). They have high accuracy and high resistance to fracture and wear. SLA uses a LASER to cure liquid resin into hardened material.

For both splints, static occlusion was designed in order to have multiple contacts between the lower buccal cusps and the surface area of the splint. Canine guidance was designed for both left and right lateral movements. Protrusion was guided by the central and lateral lower incisors. (fig. 7)

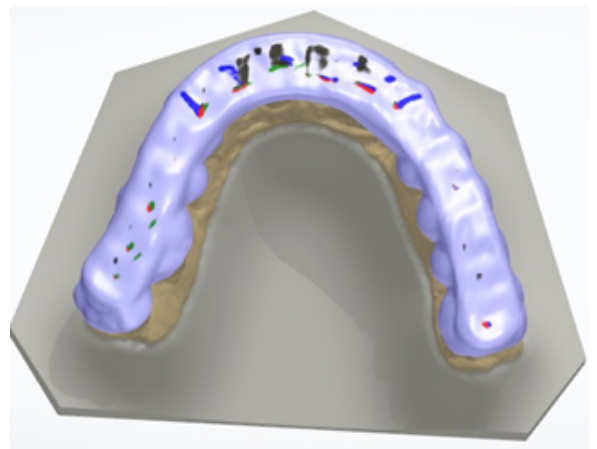

Fig. 7 Static and dynamic virtual occlusion

After the splints were printed/milled, they were checked on the plaster casts mounted in the semiadjustable articulator to see if there was any discrepancy between them and the digital model (fig. 8).

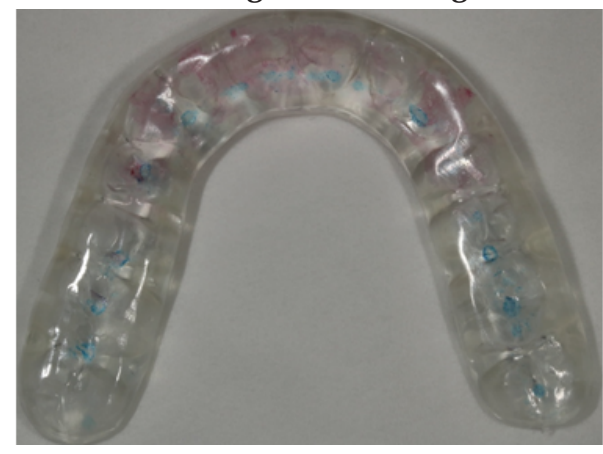

Fig. 8 Final occlusion on the splintafter finishing in semiadjustable articulator

\section{Results and discussions}

The benefits of digital splint were appreciated in terms of working time, accuracy (fit), costs and occlusion.

The mean working time for a conventional splint in the lab was about 5 hours. For a digital splint, the design took about 1 hour. The printing process took $1.5 \mathrm{~h}$, whereas the milling process took $1.15 \mathrm{~h}$. Finishing and polishing the splint took about 30 minutes for both the milled and printed splint. So, the time was slightly longer for the printed splint. But the average time for adjusting the appliance in the patient's mouth was significantly lower for the printed splint.

An important characteristic of the digital splint is the smoothness of the surface area. This feature allows a free and smooth lateral and protrusive guidance on the splint.

The fit of the digital splint over the teeth was very good, allowing insertion and removal of the splint easily, while still maintaining the friction fit. This makes the patient feel quite comfortable and is also an essential aspect in splint therapy because the stability of the splint over the teeth is mandatory. The fit of the digital splint (both printed and milled) was significantly better than of the conventional one.

When selecting the occlusion, the dental technician tried to obtain the flattest surface possible with multiple contacts with the opposing teeth. The lateral guidance was designed in order to obtain a canine guidance, whereas the protrusion was designed only on the anterior teeth, with immediate separation of the posterior teeth. Although the occlusion design was perfectly created by the dental technician, when the splint was tested in the patient's mouth, occlusal adjustments were required due to the lack of virtual facebow transfer. The lateral and protrusive guidance were exactly like they were designed in the software. The best occlusal scheme was obtained on the printed splint.

An important advantage of the printed splint as compared to the milled splint is the cost, lower for the printed splint. 
Another important advantage of the printed versus milled splint is related to the quantity of vertical dimension of occlusion (VDO) modification, meaning the thickness of the splint. When VDO alteration is important, the dimension of the PMMA disc may be insufficient to ensure all the needed occlusal characteristics. The thickest PMMA disc on the market is $25 \mathrm{~mm}$. This was not an issue in the case of printed splints.

Al-Dwairi et al. [11] tested the flexural resistance for conventional splint and for CAD CAM splint. The results showed that CAD CAM PMMA has more flexural strength and is more durable than the conventional one.

Bernsten et al. [12] compared clinically the conventional and the additive manufactured stabilization splints. The conclusions were that the patients preferred digital intraoral scanning to alginate impressions, though it was more time consuming. At the same time, the subjects reported they felt better with the CAD CAM splints.

Another study performed by Dedem et al. [13] highlighted the advantages of CAD CAM appliances: time efficient, high material quality and the possibility of manufacturing duplicate splints. Another study pointed out the benefits of the CAD CAM splint therapy: accuracy, long-term stability, biocompatibility, high resistance to fracture [14].

\section{Conclusions}

CAD CAM occlusal splints showed both good internal fit on teeth and excellent smoothness, which allowed free and uninterrupted occlusal guidance.

The costs were lower for the 3D printed splint than for the milled splint.

The printed splint can have any thickness, which is not possible in the case of the milled split, the latter being limited by the thickness of the disc.

The digital splints were found to be time-efficient. The comfort reported by the patient was rated as very good.

An important feature of the digital splints is that they can be duplicated and redone any time by either method.

\section{References}

1.OKESON JP. Management of temporomandibular disorders and occlusion. 6th Edition. St. Louis, Mosby, 2008.

2.ROCABADO M. Arthrokinematics of the temporomandibular joint. Dent Clin North Am. 1983;27(3):573-594.

3.MANFREDINI D. Current concepts on temporomandibular disorders. Quintessence Publishing Co. Ltd, 2010.

4.SLAVICEK R. The masticatory organ: functions and disfunctions. Gamma Medizinisch-wissenschaftliche Forbildungs- $\mathrm{GmbH}$, Klostenburg, 2006.

5.De LEEUW R, KLASSER G. Orofacial Pain: Guidelines for Assessement, Diagnosis and Management. $5^{\text {th }}$ ed. Chicago: Quintessence Publishing Co., Inc. 2013.

6.LAUREN, M., McINTYRE, F. A new computer-assisted method for design and fabrication of occlusal splints. American Journal of Orthodontics and Dentofacial Orthopedics 2008;133(4):S130-S135.

7.WARUNEK P, LAUREN M. Computer-based fabrication of occlusal splints for treatment of bruxism and TMD. Journal of Clinical Orthodontics 2008; 42(4):227-32.

8.DARVELL, B., Acrylic. Materials Science for Dentistry. 2018;121-142. 9.MURARIU, A, SAVIN, C, FEIER, R, BALAN, A.,. Study Regarding the Toxic Effects of Resin-based Dental Materials. Rev.Chim.(Bucharest), 67, no.9, 2016, p.1876-8.

10.CHECHERITA, L., BELDIMAN, M.A., STAMATIN, O., FOIA., L., FORNA, N.C., Aspects on Structure of Materials Used for Different Types of Occlusal Splints. Rev.Chim.(Bucharest),64, no.8, 2013,p. 864.

11.AI-DWAIRI Z, TAHBOUB K, BABA N, GOODACRE C. A Comparison of the Flexural and Impact Strengths and Flexural Modulus of CAD/ CAM and Conventional Heat-Cured Polymethyl Methacrylate (PMMA). Journal of Prosthodontics. 2018;1-9.

12.BERNTSEN, C., KLEVEN, M., HEIAN, M. AND HJORTSJ O, C. Clinical comparison of conventional and additive manufactured stabilization splints. Acta Biomaterialia Odontologica Scandinavica 2018;4(1):8189.

13.DEDEM P, TURP JC. Digital Michigan splint - from intraoral scanning to plasterless manufacturing. Int J Comput Dent. 2016;19(1):63-76.

14.EDELHOFF D, SCHWEIGER J, PRADTNER 0, TRIMPL J, STIMMELMAYR M, GUTH JF. CAD/CAM splints for the functional and esthetic evaluation of newly defined occlusal dimensions. Quintessence Int. 2017; 48(3):181-191.

Manuscript received: 28.07 .2018 